\title{
Evaluation of Methods for Cyanobacterial Cell Lysis and Toxin (Microcystin-LR) Extraction Using Chromatographic and Mass Spectrometric Analyses
}

\author{
In S. $\mathrm{Kim}^{\dagger}$, Giang Huong Nguyen, Sungyoun Kim, Jinwook Lee, and Hye-Weon Yu \\ Bio-Environmental Engineering Laboratory, Department of Environmental Science and Engineering, Gwangju Institute of Science and \\ Technology, Oryong-dong Buk-gu, Gwangju, 500-712, Korea
}

Received July, 2009 ; Accepted November,2009

\begin{abstract}
Contamination of microcystins, a family of heptapeptide hepatotoxins, in eutrophic water bodies is a worldwide problem. Due to their poisoning effects on animals and humans, there is a requirement to characterize and quantify all microcystins present in a sample. As microcystins are, for most part, intracellular toxins produced by some genera of cyanobacteria, lysing cyanobacterial cells to release all microcystins is considered an important step. To date, although many cell lysis methods have been used, little work has been conducted comparing the results of those different methods. In this study, various methods for cell lysis and toxin extraction from the cell lysates were investigated, including sonication, bead beating, freeze/thaw, lyophilization and lysing with TritonX-100 surfactant. It was found that lyophilization, followed by extraction with $75 \%$ methanol, was the most effective for extracting toxins from Microcystis aeruginosa cells. Another important step prior to the analysis is removing impurities and concentrating the target analyte. For these purposes, a C18 Sep-Pak solid phase extraction cartridge was used, with the percentage of the eluent methanol also evaluated. As a result, methanol percentages higher than $75 \%$ appeared to be the best eluting solvent in terms of microcystin-leucine-arginine (MC-LR) recovery efficiency for the further chromatographic and mass spectrometric analyses.
\end{abstract}

Keywords : Cell lysis, Cyanobacteria, Microcystin, Solid phase extraction, Liquid chromatography-mass spectrometry

\section{Introduction}

Harmful algal blooms, which usually present in stagnant water bodies, impact on water quality worldwide. Globally, the most commonly encountered cyanobacterial hepatotoxins are the cyclic peptide microcystins, which have been the cause of human and animal health hazards and even deaths. ${ }^{1)}$ Microcystins potentially inhibit the function of eukaryotic protein serine/threonine phosphatases type 1 (PP1) and 2A (PP2A), ${ }^{2)}$ which can lead to hepatocyte necrosis and hemorrhage at high levels. ${ }^{3)}$ In addition to acute poisonings, exposure to low concentrations of microcystins in drinking water can cause chronic effects in mammals due to their potent tumor promoting activity. ${ }^{3)}$ Epidemiological studies have shown that long term exposure to microcystins via drinking water supplies are associated with primary liver cancer. In 1996, for instance, the deaths of over 50 patients at a haemodialysis clinic in Brazil were attributed to microcystins, which were later identified in the clinic's water supply. ${ }^{4)}$ The increased rates of primary liver cancer in some areas of China have also been attributed to the contamination of drinking water with microcystins. ${ }^{3)}$

Of more than 80 microcystin variants identified to date, microcystin-leucine-arginine (MC-LR) has been the most extensively investigated due to its toxicity and frequent presence in cyanobacterial blooms in rivers and lakes all over the world. ${ }^{1,2)}$ Microcystin-LR is a monocyclic heptapeptide hepatotoxin, which contains seven peptide-linked amino acids, with the molecular structure cyclo - (d-alanine [1] - leucine [2] - d-MeAsp [3] - arginine [4] - adda [5] - d-glutamate [6] - mdha [7]), in which d-MeAsp is d-erythro- -methylaspartic acid, mdha $\mathrm{N}$-methyldehydroalanine and adda (2S, 3S, 8S, 9S)-3-amino-9methoxy-2, 6, 8-trimethyl-10-phenyldeca-4E,6E-dienoic acid (Fig. 1) ${ }^{5)}$ Leucine and arginine are protein amino acids, while the other five are non-protein amino acids and more or less constant between variant microcystins. MC-LR is an amphiphatic molecule. Hydrophilic functional groups, including carboxyl groups on glutamic acid and methylaspartic acid, as well as the amino group on arginine, while the Adda residue is hydrophobic.

Microcystins are intracellular secondary metabolites only produced by certain strains of cyanobacteria. ${ }^{7)}$ These toxins are synthesized within the cells and released mainly to the surrounding water when the cells are lysed or become old and leaky. ${ }^{1,2)}$ During the growth phase, microcystins are, for

\footnotetext{
${ }^{\dagger}$ Corresponding author

E-mail : iskim@gist.ac.kr

Tel: +82-62-907-3381, Fax: +82-62-970-2434
} 


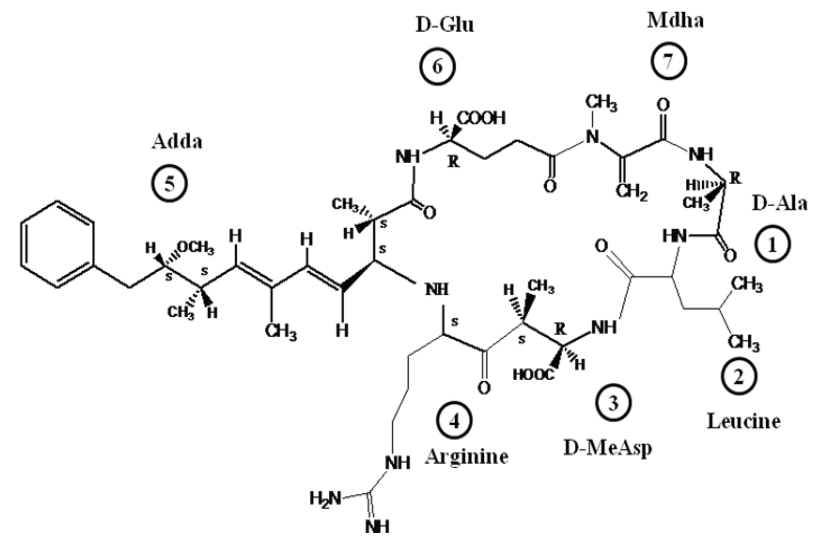

Fig. 1. The molecular structure of MC-LR.

most part, intracellular (fewer than 10 to $20 \%$ of total toxins are extracellular). ${ }^{8)}$ It is the potential chronic toxicity from microcystins that led the World Health Organization (WHO) to establish a guideline of $1 \mu \mathrm{g} / \mathrm{L}$ as a maximum concentration of total MC-LR in drinking water. ${ }^{2)}$ In addition, MC-LR was classified as a carcinogen according to the International Agency for Research on Cancer (IARC). ${ }^{9)}$ Microcystin-LR was chosen as a representative microcystin in this study because it is the most toxic and abundant variant. Moreover, the commercial availability of MC-LR standard makes it easy to gain reproducible results.

Prior to analysis of microcystin-LR, effective sample preparation steps are required. ${ }^{10,11)}$ Ass the provisional guideline level of both intracellular and extracellular MC-LR in drinking water is as low as $1 \mu \mathrm{g} / \mathrm{L}$, the outer membranes of cyanobacterial cells have to be disrupted. In the literature, several cell lysis methods that affect the extraction yield of microcystins have been used in the laboratory, including successive freeze-thaw cycles, autoclaving, sonication, heating, lyophilization, and the use of detergents, such as TritonX-100. However, there is no conclusive evidence regarding approach that performs best. ${ }^{12)}$ For example, sonication is the most commonly employed method of extracting microcystins. However, this has been found to degrade commercial MC-LR standard solutions, and prolonged use of probe sonicators should be avoided as small samples might reach high temperatures and evaporate or degrade. Also, glass fiber filters, used for biomass concentration and drying, disintegrate and release fibers, which can interfere the analysis. ${ }^{13)} \mathrm{A}$ concentration step prior to analysis is also required, as extraction of cyanobacterial cells typically results in large volumes of solvent that contain relatively dilute amounts of MC-LR. ${ }^{12)}$ Moreover, a concentration step is also required to remove the many impurities from the complex sample matrices in order to extend the liquid chromatographic column life.

In this study, the efficiency of various methods for lysing cyanobacterial cells to release intracellular toxins, and then for extracting microcystins from the cell lysates were evaluated. Isolation and concentration of MC-LR for analysis via high performance liquid chromatography coupled with electrospray ionization and a mass spectrometer (HPLC-ESI-MS), a widely accepted technique for determining MC-LR, were also investigated.

\section{Experimental Methods}

\subsection{Chemicals and Reagents}

All chemicals and reagents were of either HPLC or analyticalreagent grade. The microcystin-LR standard was purchased from Alexis Biochemicals (San Diego, CA, USA). HPLC grade water, acetonitrile and methanol were purchased from Fisher Scientific Korea Ltd. (Seoul, Korea). Formic acid was purchased from Fluka Chemical Corp (Milwaukee, WI, USA). Deionised water was obtained by passing tap water through a Minipore system (Simpak Kit 1; Millipore, Paris, France) with the resistance $>18.2 \mathrm{M} \Omega /$ $\mathrm{cm}$. BG11 powder was obtained from Fluka BioChemika (Buchs, Switzerland).

\subsection{Cultivation of Microcystis aeruginosa}

Microcystis aeruginosa strain (NIER-10111) was obtained from the National Institute of Environmental Research (NIER), Korea and cultivated in $\mathrm{BG} 11$ medium at $20^{\circ} \mathrm{C}-25^{\circ} \mathrm{C}$ under continuous illumination (2,500 - 3,000 lux, measured with a digital Lux meter DX-100 [INS Enterprise Co., Ltd., Taipei, Taiwan]) and stirring at a rate of $100 \mathrm{rpm}$. Cells were harvested after approximately 30 days growth and used as material for investigating the efficiencies of various cell lysis methods.

\subsection{Efficiency of Cell Lysis Methods}

Different methods for disrupting $M$. aeruginosa cells were studied, including freeze/thaw, sonication, lyophilization, bead beating, and $1 \%$ TritonX-100 surfactant, as described in Table 1 . The numbers of cyanobacterial cells, both before and after treatment, were measured by direct microscopy counting. Cells were injected into the counting chamber of an improved Neubauer hamocytometer (Marienfeld, Germany), with images captured by a LSM5 (Zeiss, Germany) inverted confocal laser scanning microscope (CLSM), equipped with a PC, using the LSM software (PASCAL) to control all the system components. Imaging was achieved using a C-Apochromat $40 \times$ objective. The efficiency of each cell lysis method was calculated via dividing the number of disrupted cells after treatment by the initial number of cells; this was then expressed as a percentage.

Table 1. Methods for Microcystis aeruginosa cell lysis

\begin{tabular}{ll}
\hline Method & Description \\
\hline Sonication & $\begin{array}{l}\text { Sonicate for } 30 \text { min using a Branson } \\
\text { model } 5,510 \text { ultrasonic cleaner. }\end{array}$ \\
\hline Lyophilization & $\begin{array}{l}\text { Freeze the sample before lyophilization. } \\
\text { Lyophilize for } 3 \text { days using a Freeze-dryer. } \\
\text { Re-suspend to the initial volume with } \\
\text { either deionised water or } 75 \% \text { methanol. }\end{array}$ \\
\hline Bead beating & $\begin{array}{l}\text { Bead beat using either } 0.1 \text { or } 0.5 \mathrm{~mm} \\
\text { beads, the remaining volume will contain } \\
\text { the sample. Bead beat at } 4,200 \text { rpm for } 3 \\
\text { min }(1 \text { min "ON," then } 1 \text { min “OFF" and } \\
\text { cooling of the sample with ice, cycling } 3 \\
\text { times). }\end{array}$ \\
\hline Freeze/thaw & $\begin{array}{l}\text { Freeze at }-70^{\circ} \mathrm{C} \text { for } 10 \text { min, then thaw at } \\
37^{\circ} \mathrm{C} \text { for } 5 \text { min. Repeat } 3 \text { times. }\end{array}$ \\
\hline TritonX-100 & $\begin{array}{l}\text { TritonX-100 was mixed with cell culture } \\
\text { solution to a final concentration of } 1 \% .\end{array}$ \\
\hline
\end{tabular}




$$
E(\%)=\left(C_{i}-C_{f}\right) / C_{i} \times 100 \%
$$

where, E(\%) : Efficiency (\%) of a cell lysis method

$$
\begin{aligned}
\mathrm{C}_{\mathrm{i}} \text { : The initial concentration of M. aeruginosa cells } \\
\text { (i.e. before cell lysis) } \\
\mathrm{C}_{\mathrm{f}} \text { : The final concentration of M. aeruginosa cells } \\
\text { (i.e. after cell lysis) }
\end{aligned}
$$

\subsection{Recovery Efficiency of MC-LR through a C18 Solid Phase Extraction Cartridge}

Microcystin-LR was concentrated on a C18 Sep-Pak/Vac 3 cc, $500 \mathrm{mg}$ solid phase extraction (SPE) cartridge (Waters Corporation, Milford, MA, USA) using an extractor and vacuum pump (Waters Corporation) at a flow rate of $5 \mathrm{~mL} / \mathrm{min}$. The procedure can be briefly described as follows: $3 \mathrm{~mL}$ of $300 \mu \mathrm{g} / \mathrm{L}$ MC-LR, prepared in either absolute methanol or $15 \%$ methanol, was applied to a preconditioned SPE cartridge. The preconditioning step included washing with $10 \mathrm{~mL}$ of methanol, followed by $10 \mathrm{~mL}$ of deionised water. The cartridge was then washed with $10 \mathrm{~mL}$ of $15 \%$ methanol and $10 \mathrm{~mL}$ of deionised water to remove the undesired impurities. Finally, the target peptide was eluted using $3 \mathrm{~mL}$ of different percentages of methanol, ranging from 55 to $95 \%$ at $10 \%$ increments. All eluted fractions from loading, washing and eluting steps were collected and analyzed by HPLC-ESIMS, which was performed using a Waters Alliance 2695 HPLC pump coupled to an electrospray (ESI) triple stage quadrupole Quattro micro (Waters Micromass; Waters Corporation) mass spectrometer (Fig. 2).

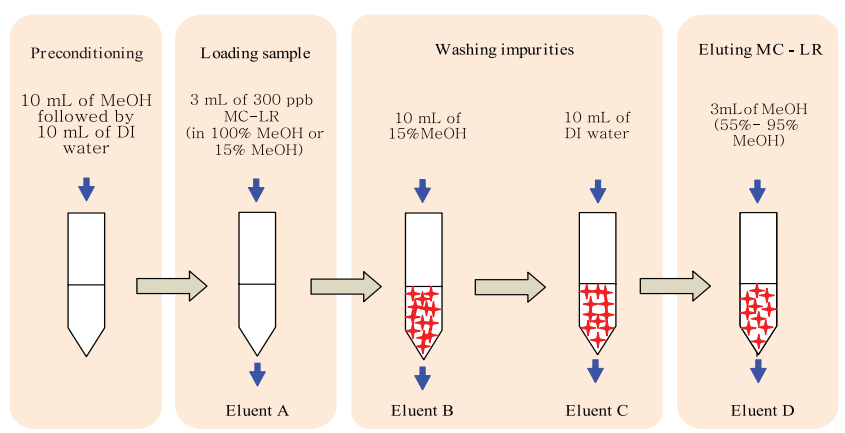

Fig. 2. The procedure for concentrating MC-LR.

The HPLC system, consisting of a separation module (Waters 2695; Waters Corporation), an autosampler (Waters Alliance 2695; Waters Corporation) and a photodiode array detector (Waters 996; Waters Corporation), was controlled by MassLynx 4.1 software. The chromatographic separation was performed on a SunFire C18 column (2.1 mm i.d., $150 \mathrm{~mm}$ length, $3.5 \mu \mathrm{m}$ pore size) using a mobile phase of acetonitrile and water containing $0.1 \%$ formic acid at a flow rate of $0.3 \mathrm{~mL} / \mathrm{min}$. The gradient conditions for a standard sample were: 0 min: $25 \%$ organic, $4-5$ min: $75 \%$ organic, $10 \mathrm{~min}: 25 \%$ organic. The volume of sample injected was $10 \mu \mathrm{L}$.

An electrospray (ESI) triple stage quadrupole Quattro micro (Waters Micromass; Waters Corporation) mass spectrophotometer was used to determine the molecular mass of MC-LR. Ions were formed in the positive ionization mode $[\mathrm{M}-\mathrm{H}]^{+}$. The ion spray voltage was set at $3.5 \mathrm{kV}$, the source temperature at $150^{\circ} \mathrm{C}$ and the desolvation temperature at $350^{\circ} \mathrm{C}$.

\section{Results and Discussion}

\subsection{Efficiency of Cell Lysis Methods}

The cell lysis process was carried out to release intracellular materials, assisting the more efficient extraction of toxins of interest. Fig. 3(a) shows a visual image of the laboratory batch culture of $M$. aeruginosa NIER $10111\left(\mathrm{OD}_{660}=0.36\right.$ after 30 days growth) used as a material for evaluating several cell lysis methods, while Figs. 3(b) and (c) showed the differential interference contrast (DIC) images of this cyanobacterium strain on a glass slide and on a square cell of a counting chamber (haemocytometer), which were taken using a CLSM. M. aeruginosa cells varied from 3 to $7 \mu \mathrm{m}$ in diameter and were present in an isolated form under laboratorial conditions.

(a)

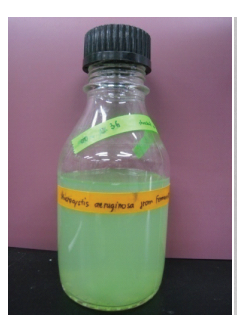

(b)

(c)

Fig.3. M. aeruginosa strain NIER-10111 after 30 days growth, incubated between $20^{\circ} \mathrm{C}-25^{\circ} \mathrm{C}$, illumination $=2,500-3,000$ lux, shaking rate $=$ $100 \mathrm{rpm}$. (a) Visual image of laboratory batch culture. (b) Differential interference contrast (DIC) image of cells on glass slide by confocal laser scanning microscope (CLSM; scale bar of $10 \mu \mathrm{m})$. (c) DIC image of cells on a haemocytometer cell by CLSM.

Before being lysed, the $M$. aeruginosa cell number was determined as $5.7 \pm 0.9 \times 10^{6}$ cells $/ \mathrm{mL}$ via direct microscopic counting on the haemocytometer. After the treatments using various cell lysis methods, as described in section 2.3 , the efficiencies of those methods were calculated to be $73.3 \pm 0.9 \%$ for sonication in a water bath, $92.2 \pm 0.5 \%$ for lyophilization followed by extraction with deionised water, $98.4 \pm 0.7 \%$ for lyophilization followed by extraction with $75 \%$ methanol, $98.2 \pm 0.3 \%$ for bead beating using $0.1 \mathrm{~mm}$ glass beads, $99.2 \pm 0.3 \%$ for bead beating using $0.5 \mathrm{~mm}$ glass beads, $19.4 \pm 1.2 \%$ for three sequential freeze/ thaw cycles, and $37.3 \pm 1.4 \%$ for $1 \%$ TritonX-100 surfactant, as depicted in Fig. 4.

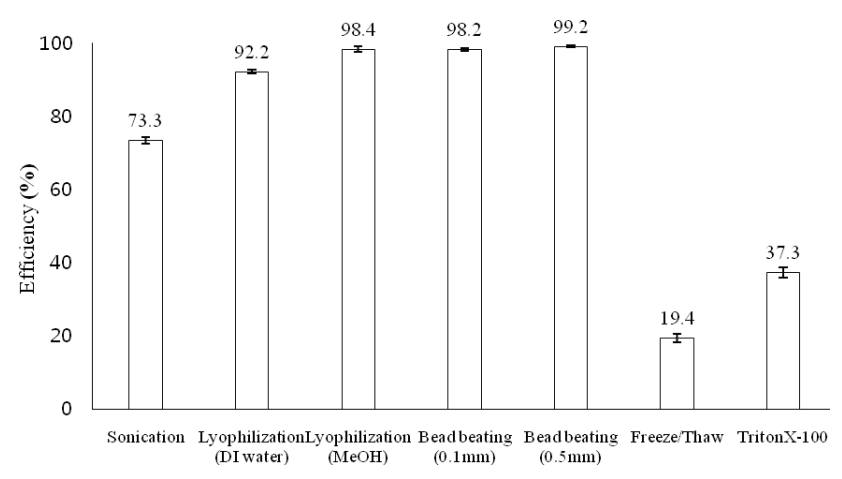

Fig.4. Efficiency of methods for M. aeruginosa cell lysis ( $\mathrm{n}=3$, error bars are the standard deviation of the mean). 
The results obtained showed that lyophilization followed by the extraction of freeze-dried cyanobacterial mass with $75 \%$ methanol, the best solvent for microcystins extraction reported in literature, was sufficient to disrupt the $M$. aeruginosa cell membranes. Although bead beating appeared to be an excellent method for cell lysis, as the efficiencies obtained were over $98 \%$ for both diameters of glass beads, it was possible that some of the microcystins were degraded due to the high operating temperature and by becoming attached onto the bead surface, leading to the loss of microcystins in the extract. Freezing the cells at $-70^{\circ} \mathrm{C}$ for $10 \mathrm{~min}$ and then thawing at $37^{\circ} \mathrm{C}$ for 5 min did not show sufficiency breaking down of the cyanobacterial cell membranes; even though this method has been reported as a conventional cell lysis method in many documents. The use of TritonX-100 as a chemical detergent for lysing the cells did not disrupt many of the M. aeruginosa cell membranes. Moreover, it was quite time and labor consuming to remove the surfactant from the cell lysates in the further clean-up step. Therefore, for extracting endotoxins from M. aeruginosa cells, lyophilizing followed by vortexing the biomass in $75 \%$ methanol was chosen in this study..

\subsection{Recovery Efficiency of MC-LR through a C18 Solid Phase Extraction Cartridge}

A calibration curve of the MC-LR precursor ion $(\mathrm{m} / \mathrm{z}=995.6)$ was plotted to evaluate the linearity of the HPLC-ESI-MS method (Fig. 5(a)). A lower limit of quantification of 1 ppb was obtained, with a correlation coefficient $\left(\mathrm{r}^{2}\right)$ value higher than $99 \%$. The assay proved to be linear and acceptable.

\subsubsection{Loss in Loading and Washing Steps}

An experiment was carried out with MC-LR variant to determining the potential losses during the loading and washing steps of the concentration process using the solid phase extraction method. Fig. 5(b) showed the losses of MC-LR during the loading and washing steps when the loaded MC-LR solutions contained different percentages of methanol. Applying MC-LR prepared in high percentage of methanol $(100 \%)$ led to considerable losses during the loading (elutant A: $15.1 \pm 0.9 \%$ ) and washing steps (elutant B: $5.8 \pm 0.0 \%$, and C: $3.9 \pm 0.1 \%$ ), approximately $24.8 \pm 1.0 \%$ in total. Therefore, as the losses were under detection range in all steps, the MC-LR lost appeared to be insignificant when prepared in a low percentage of methanol $(15 \%)$ prior to application to the C18 SPE cartridge. It was obvious that the percentage of methanol in the extract loaded onto the C18 SPE cartridge was a significant factor affecting the recovery efficiency of MC-LR. Therefore, reducing the percentage methanol from 75 to $15 \%$ was required after the solvent extraction step, which was achieved using a rotary evaporator.

\subsubsection{Recovery Efficiency of Eluting Step}

Fig. 5(c) showed the recovery efficiency of MC-LR when this toxin was eluted with different concentrations of methanol using a solid phase extraction method. It was found that employing methanol with percentages higher than $75 \%$ provided relatively similar recovery efficiencies for MC-LR, i.e. $85.0 \% \pm 10.0 \%$ with $75 \%$ methanol, $83.7 \% \pm 7.4 \%$ with $85 \%$ methanol, and $85.4 \% \pm 9.8 \%$ with $95 \%$ methanol. A low concentration of methanol; $55 \%$, was unable to elute MC-LR at a detectable concentration because MCLR is relatively hydrophobic. Since $75 \%$ methanol offers the ability to extract analytes with a wide range of polarities, as reported in many previous publications, this percentage was chosen for further experiments to observe the recovery efficiency of MC-LR together with many other MC variants.

(a)

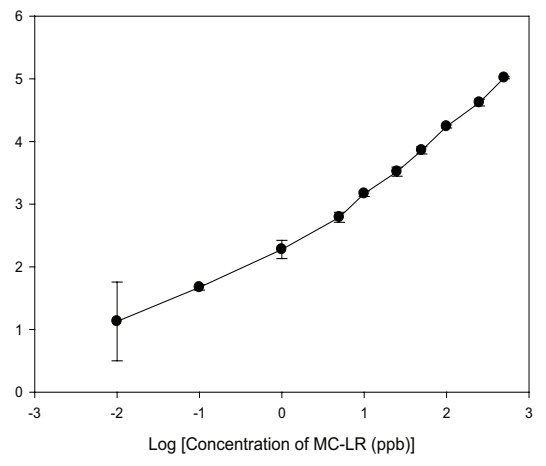

(b)

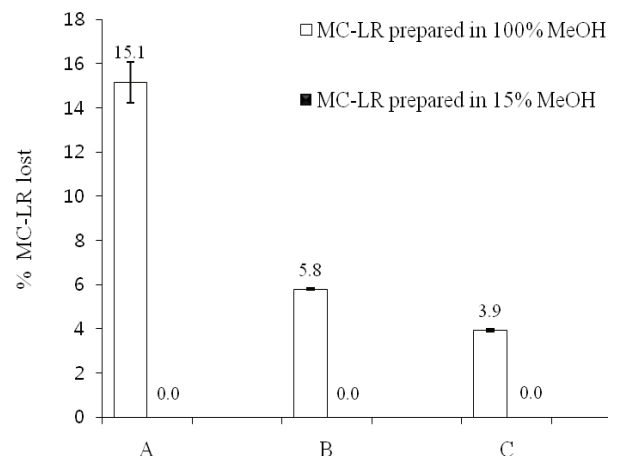

(c)

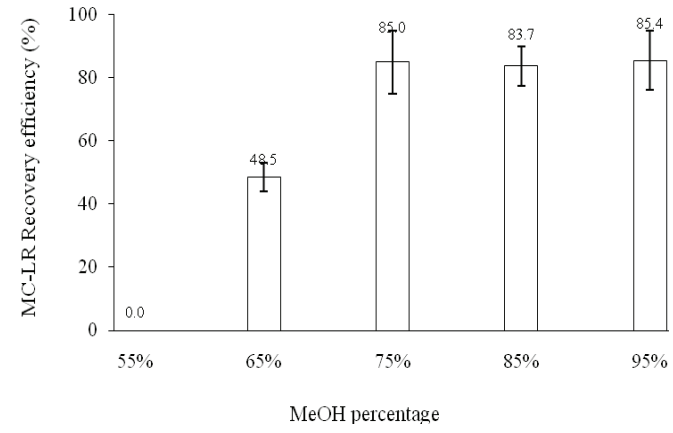

Fig. 5. (a) The calibration curve of MC-LR standard ( $\mathrm{m} / \mathrm{z}=995.6)$, (b) Loss of MC-LR during loading (A) and washing (B and C) steps of the solid phase extraction method, and (c) Recovery efficiency of MC-LR during the elution step of the solid phase extraction method. ( $n=3$, error bars are the standard deviation of the mean). 


\section{Conclusions}

Of all the cell lysis methods evaluated, lyophilization followed by extraction with $75 \%$ methanol yielded the best result for releasing the intracellular toxin and extracting the target toxin from the cell lysates. The conventional C18 SPE method was appropriate for isolating and concentrating MC-LR containing only low concentrations of methanol, which was then eluted with methanol percentages higher than $75 \%$. Therefore, after extracting MC-LR from the biomass, dilution of the extraction solvent methanol to a low concentration (approximately 15\%) is recommended before loading onto the C18 SPE cartridge.

\section{Acknowledgements}

This research was supported by a grant (07SeaHeroA01-01) from the Plant Technology Advancement Program, funded by the Ministry of Land, Transport and Maritime Affairs of the Korean Government.

\section{References}

1. Sangolkar, L. N., Maske, S. S., and Chakrabarti, T., "Methods for determining microcystins (peptide hepatotoxins) and microcystin-producing cyanobacteria," Water Res., 40(19), 3485-3496 (2006).

2. World Health Organization, Guidelines for drinkingwater quality: incorporating 1st and 2nd addenda, Vol.1, Recommendations, 3rd ed., WHO, Geneva (2008).

3. McElhiney, J. and Lawton, L. A., "Detection of the cyanobacterial hepatotoxins microcystins," Toxicol. Appl. Pharmacol., 203(3), 219-230 (2005).

4. Yuan, M., Carmichael, W. W., and Hilborn, E. D., "Microcystin analysis in human sera and liver from human fatalities in Caruaru, Brazil 1996," Toxicon, 48(6), 627-640 (2006).

5. Dai, M., Xie, P., Liang, G., Chen, J., and Lei, H., "Simultaneous determination of microcystin-LR and its glutathione conjugate in fish tissues by liquid chromatography-tandem mass spectrometry," J. Chromatogr. B Analyt. Technol. Biomed. Life Sci., 862(1-2), 43-50 (2008).

6. Vesterkvist, P. S. M. and Meriluoto, J. A. O., "Interaction between microcystins of different hydrophobicities and lipid monolayers," Toxicon, 41(3), 349-355 (2003).

7. Juttner, F. and Lüthi, H., "Topology and enhanced toxicity of bound microcystins in Microcystis PCC 7806," Toxicon, 51(3), 388-397 (2008).

8. Best, J. H., Eddy, F. B., and Codd, G. A., "Effects of purified microcystin-LR and cell extracts of Microcystis strains PCC 7813 and CYA 43 on cardiac function in brown trout (Salmo trutta) alevins," Fish Physiol. Biochem., 24(3), 171-178 (2001).

9. Grosse, Y., Baan, R., Straif, K., Secretan, B., El Ghissassi, F., and Cogliano, V., "Carcinogenicity of nitrate, nitrite, and cyanobacterial peptide toxins," Lancet Oncol., 7(8), 628-629 (2006).

10. Lee, J. W., Yu, H. W., and Kim, I. S., "Application of quantumdot nanocrystals for cyanobacterial toxin-microcystin detection," J. Korean Soc. Water Qual., 23(5), 705-711 (2007).

11. Yu, H. W., Lee, J., Kim, S., Nguyen, G. H., and Kim, I. S., "Electrochemical immunoassay using quantum dot/antibody probe for identification of cyanobacterial hepatotoxin microcystin-LR," Anal. Bioanal. Chem., 394(8), 2173-2181 (2009).

12. Lawton, L. A. and Edwards, C., "Purification of microcystins," J Chromatogr. A, 912(2), 191-209 (2001).

13. Spoof, L., Vesterkvist, P., Lindholm, T., and Meriluoto, J., "Screening for cyanobacterial hepatotoxins, microcystins and nodularin in environmental water samples by reversedphase liquid chromatography-electrospray ionisation mass spectrometry," J. Chromatogr. A, 1020(1), 105-119 (2003). 